\title{
PHANTOM LIMBS
}

\author{
BY \\ W. R. HENDERSON and G. E. SMYTH \\ Leeds \\ Manchester \\ Formerly No. 1 Mobile Neurosurgical Unit, R.A.M.C.
}

(RECEIVED JANUARY 26, 1948)

\section{INDEX}

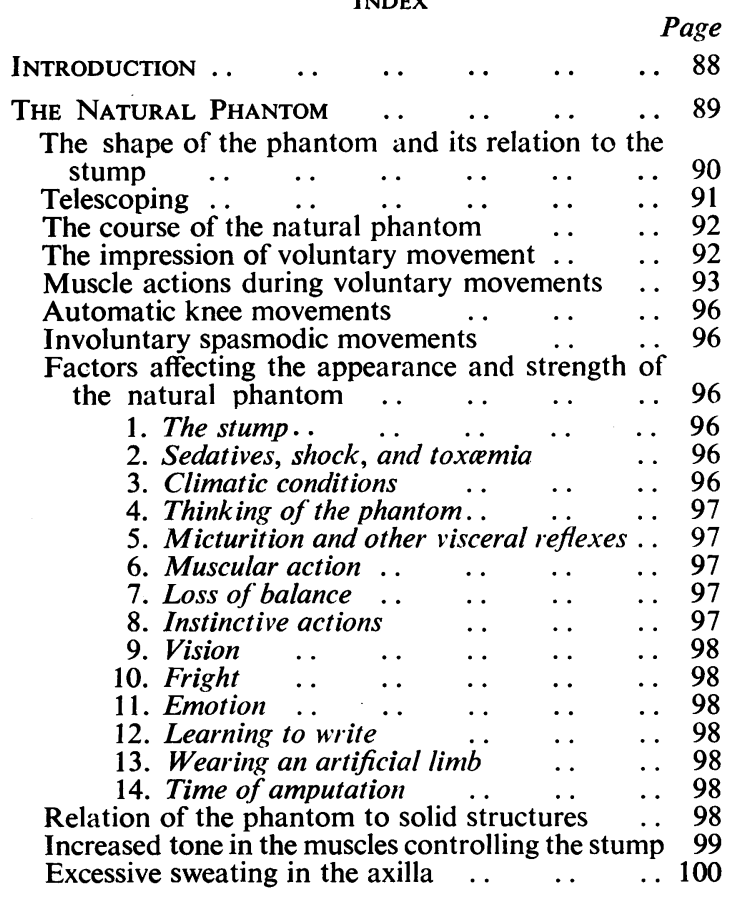

The Neuroma Sensation $\ldots$.. $\ldots$.

Modification of the Phantom from a Higher

Cerebral Level $\ldots$.. $\quad . \quad$.

Superadded sensations of pre-amputation impres-

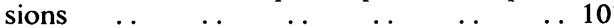

The possible modifying influence of pre-amputa$\begin{array}{llllllll}\text { tion posture } & \ldots & \ldots & \ldots & \ldots & \ldots & 102\end{array}$

Suppression of part of the natural phantom $\quad \ldots 102$

The occurrence of unnatural attitudes or abnormally free movements $\quad . \quad \ldots \quad \ldots \quad \ldots 103$

Submergence of the natural phantom by an overlay of disagreeable manifestations $\ldots \quad \ldots \quad \ldots 103$

SECONDARy Stump Operations $\quad \ldots \quad$.

Double AMPUTATIONS $\quad$.

Minor AmpUtATIONS ..

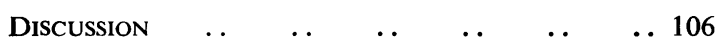

$\begin{array}{llllllll}\text { SUMMARY } & \ldots & \ldots & \ldots & \ldots & \ldots & \ldots & 111\end{array}$

\section{INTRODUCTION}

The various phenomena which may appear in a phantom limb are so puzzling and their elucidation so beset with difficulty that it is with some hesitancy that we approach the problem. The difficulty is not lessened by the fact that several penetrating studies have appeared recently, notably by Lhermitte (1939) and Riddoch (1941). Close contact, however, with many amputation cases over a long period provided an opportunity to study limb phantoms, and the excuse for this paper must be that our conclusions are in some important respects at⿱亠े $\infty$ variance with those currently held. This study is $\frac{0}{\mathrm{\omega}}$ 을 not a comprehensive review but is limited to those aspects which seem to us to be the more important in illuminating what may be called the physiologyo of the phantom.

Material.-The observations were made in pris oner-of-war hospitals and camps in Germany in the years 1940-45. The patients were all men of military age, and amputation was nearly always a sequel to gun-shot wounds. The series was, therefore, fairly uniform and the circumstances of a prisoner-of-war life alike for all.

Some three hundred major and many minor amputations were seen. Amputation had been done usually within the first week or two after wounding, but sometimes not for several months and occasionally as late as twelve to eighteen months. The first ninety-eight cases came under our care six months after wounding in 1940 . Thereafter, new cases arrived periodically from other campaigns, and in most of them amputation had already been done weeks or months earlier. The majority of observations began, therefore, when stump and phantom had reached a relatively stable stage. Other cases were seen in the early days, and a few studies were made both before and after amputation. Most of the men remained under observation until repatriated one to three years later, thus allowing study of the phantom at intervals through the more important changes in its evolution. 
We were much impressed by the men's co-operation in describing their impressions, and somewhat surprised by their ready acceptance of, and indifference to, the phantom, and by its negligible influence on their outlook. Repeated questioning had no effect in focusing their attention on this curious phenomenon, neither had the irksomeness of captivity, nor the close contact of so many victims. All were in the same plight, their morale was high, and they were eager to overcome the disability-all probably important factors in relation to the strikingly low incidence of painful phantom. It is possible that in other circumstances, for example in later life, after industrial and civilian accidents involving compensation, or when vascular disease necessitates amputation, there is more likelihood of pain or other disagreeable features appearingepiphenomena, as we believe-which may come to dominate the usual mild sensation of the missing part.

It was found that the men varied greatly in their ability to describe the phantom, and also, apparently, to feel it ; and in this respect they fall roughly into three groups. (1) A minority, about 10 per cent., of men with alert minds and well marked phantoms who had made original observations (for example in relation to voluntary movement) and could describe their impressions clearly. These were the most instructive cases and provided important clues for our further inquiries. The occasional man with vivid descriptive powers and an hysterical overlay is, with experience, easily recognized.

(2) The majority, about 80 per cent., in whom the typical phantom had been so mild and unobtrusive that little thought had been given to it, and it was described in very general terms. These cases gave the true picture of the average phantom, which is painless and never complained of, and they provided statistical confirmation of the main conclusions acquired from individuals in the first group. (3) The remaining 10 per cent., men of poor intelligence, were unable to describe their impressions or to amplify the vague statement that they felt the hand ; they provided no useful information.

Only very few, about 2 per cent., asserted they had never felt a phantom. There was no discoverable reason for this absence of what appears to be a natural phenomenon, but it is perhaps remarkable that more did not refuse to believe what they themselves called "imagination" (a notion which is belied by the remarkably similar descriptions of its manifestations); one man, a Scot, steadfastly asserted that his hand was off, that was that, and he refused to believe such nonsense ! Officers, on the whole, tended to confuse the issue by overdescribing their impressions, and to experience overlay sensations; their descriptions were less useful than the men's simple statements.

Our detailed case notes were unavoidably lost in Germany at the end of the war. This paper has, therefore, been compiled from memory and is merely an outline of the main facts, without, unfortunately, the prepared statistics and illustrative cases and much detail which cannot be recalled clearly. It is, too, a pity that the men's own words, which are the essence of such a subjective study as this, have been lost. Nevertheless, our conclusions are perhaps of interest, more particularly as they were arrived at without much previous experience of the subject and without preconceived ideas derived at second hand.

Scope of Study. - This falls conveniently under three headings: (1) a description of the more important manifestations in the phantom, and a suggested simple classification of the phenomena : (2) a consideration of the possible significance of these phenomena in relation to the physiology of the nervous system; (3) the observed effects of various peripheral operations (for example, neurectomy, re-amputation), and the indications, as regards the phantom, for operation on the stump.

These three approaches, descriptive, physiological, and surgical, are partly interwoven. The descriptive aspect is the most important, and before speculating on the genesis of the phantom or considering surgical treatment it is necessary to appreciate that three fundamentally different types of sensation may occur : (1) the basic tingling of what we call the " natural phantom," which, as will presently appear, is assumed to have an organic basis at a "middle level " (sensori-motor cortex) in the central nervous system; (2) various additional and sometimes bizarre sensations, presumably of psychogenic origin, determined by activity at " highest levels" of the nervous system ; (3) a simpler " lowest level " pins and needles sensation excited by mechanical stimulation of a neuroma in the stump. Pain resembling, if not identical with, causalgia may rarely occur in the stump or phantom; we do not recollect more than three or four such cases. The differentiation of these various sensations is an important aim of this paper, but we have also speculated to some extent on their significance.

\section{THE NATURAL PHANTOM}

We wish to stress the fundamental importance of the " natural phantom," by which term is meant the almost invariable appearance in consciousness of the missing part. It is a positive sensation, usually described as tingling, or numbness, which is not painful and, indeed, is often referred to as 
" quite pleasant." The initial separating off of this almost universal, basic sensation is important, for if it is included together with other bizarre or unpleasant qualities which may appear there is danger of confusing qualities which have little in common and whose anatomical substrates in the nervous system are probably different.

This sensation is always stronger than any vague awareness of an intact limb (which, normally, is not consciously felt, at least as a positive sensation). The tingling presumably has an organic basis and depends upon impulses entering at a conscious level. Occurring at first spontaneously, the patient can also, for a time, induce the phantom sensation by merely thinking about it, but in certain circumstances it appears without his thoughts being directed to it.

The form of the phantom is not haphazard but conforms more or less closely to a common pattern which, however, does not reproduce all the amputated parts. The periphery of the limb, such as the hand or foot, and especially the digits, is the strongest and most enduring portion and may be the only part ever to appear, even after removal of a whole limb. The elbow or knee is sometimes present, the forearm or leg rarely, and the arm and thigh almost never.

The hand, as the patient often calls it, when considered in more detail, frequently consists only of the digits, which can usually be identified individually, even if represented only by the terminal pads - or knuckles, until they disappear ; but the palm, sometimes the wrist (occasionally quite strong), and less often the dorsum of the hand may also appear. As regards the digits, the thumb and index, especially the thumb, are almost always the strongest and most enduring; and in the digits the most noticeable parts are the pads and sometimes the joints. The little finger is sometimes stronger than the middle.

In the foot the same general pattern is found, with emphasis on the great toe, while the second toe is intermediate and the last three toes are very weak, often described as an ill-defined mass, or they may be entirely absent. The instep and heel are moderately strong when present, but the ankle is weak, and the dorsum rarely appears. The forefoot and instep are thus the most obtrusive parts.

The phantom therefore represents only islands and not the whole of the missing limb, and it is usually realized that there are gaps which cannot be felt. Dull, unobservant patients may say they can feel the whole limb, but, when questioned closely, admit they have no positive sensation of the entire limb. Although a well developed phantom is almost always strongest in the digits, there is no strictly progressive diminution in its strength as the stump is approached, for, as we have seen, the digits are not of equal intensity, the elbow is stronger than the forearm, and the palm is sometimes weaker than the wrist. Upper-limb phantoms are, statistically, considerably stronger and more enduring than those of the lower limb. The phantom thus appears to consist primarily of those parts which have the most extensive representation in the cerebral cortex, especially the digits and the joints, but other parts may appear in very vivid phantoms, particularly in the early stages.

\section{The Shape of the Phantom and its Relation to the Stump}

No matter how little of the missing limb appears in the phantom and how extensive the gaps, the patient has no difficulty in estimating where the various parts would be if he could feel them, their size and distance from the stump. This faculty enables him to orientate the different parts to each other and to the stump and he can readily demonstrate their attitude on the normal limb; for example, if he feels only the terminal phalanges of the digits after a shoulder disarticulation he can fit in all the unperceived missing parts and can estimate the angle which the several joints would occupy.

The phantom is correctly aligned to the stump with which it moves. In a shoulder disarticalation it moves with the scapula and in a hip disarticulation it is in line with the trunk. Its movement is not impeded by any interposed solid body, through which it can pass without any feeling of obstruction.

The hand usually assumes an attitude of rest, with the digits slightly flexed and abducted, as if holding a large ball. Sometimes the fingers are semiflexed, and rarely they are fully flexed making a clenched fist. The thumb is occasionally adducted and felt to be in contact with the index. The wrist is usually straight. The digits are never straight or hyperextended in an uncomplicated phantom; the significance of occasional bizarre attitudes will be referred to later. The hand is rarely in the position it occupied at the time of wounding. Occasionally the fingers reappear in a different attitude, for example either more or less flexed ; and the patient usually says that "the nerves feel more relaxed when the hand is open " at rest, but " feel tightened up when the hand is closed."

The elbow angle is variable. In rather more than half it is flexed at a right angle with the forearm across the front of the chest ; in others it is in full extension with the hand down by the side; while, occasionally, it is fully flexed across the upper chest, or partly extended across the abdomen. The forearm is semipronated. 
As regards the lower limb, the foot and toes are in the normal position of rest. In at least two-thirds the knee is extended ; in the remainder it is usually flexed to a right angle, but occasionally it is partly or fully flexed.

In fact, the posture of the majority of upper and lower limb phantoms recalls that of hemiplegia. The attitude of the hand or foot is not affected by the level of amputation nor, after shoulder disarticulation, is the angle of the elbow affected by the total absence of its controlling muscles.

\section{Telescoping}

This curious phenomenon, in which the digits gradually approach the stump and finally become attached to it, is so common that it must be regarded as a property of the natural phantom. It is associated with, and presumably results from, the general weakening of the phantom. The gaps which remain after the weaker parts have faded out of consciousness (or never appeared in it) retain their normal size for some time before becoming gradually smaller, and even then they do not, as a rule, disappear suddenly. This gradual shrinkage and elimination results in telescoping. It is a diffuse process, affecting most of the limb simultaneously, and not progressively from the stump towards the periphery, and is obviously a further stage of the fading out of the weaker parts.

The time at which shortening begins and the period taken for its completion are variable. The digits may reach the stump in a month or two after amputation or only after several years. The time taken does not appear to depend on the level of amputation, that is, the distance to be transversed by the digits, but, on the whole, it is quicker in the lower limb : a rough average is one to one and a half years for thigh amputations and two to two and a half years for arm amputations. This significant difference is probably related to the relative weakness of lower-limb phantoms. Although the rate of telescoping is not related to the actual distance traversed, it appears to be influenced by the strength of the phantoms of the disappearing segments as well as that of the digits. Thus telescoping was completed in thigh and leg amputations at about the same time (if anything quicker in the former, probably because it is more easily appreciated), but there is a considerable difference in the upper limb : by two years it was complete in almost all lower-limb amputations, in 80 per cent. of forearm, in only 50 per cent. of arm amputations, and in none of the nine shoulder disarticulations although it had begun in all.

Telescoping affects the long segments (proximal and distal) * gradually and simultaneously, but the digits retain their normal size much longer, hence a partly telescoped phantom is often appreciated as a "baby arm with a hand of normal size," or the disproportion may be described by saying the "fingers have become very large." There is a striking difference between the toes and fingers in a that the latter are much stronger and retain their outline much longer after telescoping. The fingers are generally normal in size on reaching the stump and can almost always be identified individually; in the lower limb usually only the great toe remains but has often lost its form and is merely a vague tingling, the other toes having already disappeared or consisting only of an indefinable conglomerate mass.

As a right angle (right) arm phantom becomes smaller the hand gradually retreats from the left side of the abdomen towards the right breast. The hand still retains its horizontal position even when it is just at the stump and the patient appreciates the small space occupied by the elbow and wrist ; then when all has gone except the digits these change direction on becoming attached to the stump and usually hang straight down, but sometimes they curl around the end of the stump as if grasping it. The thumb and great toe are always on the medial side. In at least 10 per cent. of amputations the phantom fingers retract within the lower end of the stump where they can still be identified and sometimes moved, but the much weaker toes very rarely do this.

Observant patients are usually aware of the gradual telescoping, especially in the upper limb; it is better appreciated after high amputations and when the elbow or knee is flexed-" when I think about it I realize it is short." A poor witness, however, often fails to notice the shortening, even when asked about it, until he suddenly realizes the digits are at the stump.: Occasionally, a phantom, especially when only partly telescoped, will lengthen temporarily in response to a strong intensifying stimulus (for example fright) or climatic change, so that the shortening is not strictly irreversible, and a part which had apparently been completely obliterated can reappear, but this ceases to happen soon after telescoping is complete. In rare instances no shortening occurs, especially when severe pain or psychogenic manifestations are present.

The phantom, then, evidently reflects the relative importance of cerebral representation of the different parts of the limb. The loss of anatomical proportion in the telescoping phantom makes it unlikely that appreciation of the phantom depends

* The terms proximal and distal amputation or segment of the limb are used to indicate the arm or thigh and forearm or leg respectively. 
upon any visual impression of the body scheme. The descriptions of the telescoping phantom call to mind Penfield and Boldrey's (1937) homunculus depicting cortical representation; but we would make the index finger and perhaps the wrist larger than theirs.

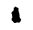

\section{The Course of the Natural Phantom}

The phantom is an immediate consequence of amputation and is often perceived when the patient awakens from the anæsthetic; there is nothing unnatural about the sensation and at first the patient may have the impression that the expected amputation has not been done until he discovers that the part, which can be felt so vividly, is absent. At first the phantom is present almost continuously, and some patients, disappointed at losing the limb, may, for a time be obsessed by this strange phenomenon. After a few weeks the tingling becomes weaker, recurs less frequently, and is noticed spontaneously only a few times during the day for perhaps ten or twenty minutes, usually when the patient has nothing to do or think about and especially in the evening when lying quietly before going to sleep after "lights out." It does not prevent or disturb sleep, and is rarely noticed when he is actively occupied.

During the next six to twelve months (it may be a much shorter or longer period) the phantom weakens considerably and its spontaneous appearances become less frequent until it is noticed only once or twice a week, although it can still be induced momentarily by thinking about it. Later it cannot be voluntarily felt (except in distal amputations when it can be induced for -a further period by voluntarily moving the digits, as described later). Finally it may continue to appear spontaneously on rare occasions, chiefly in the winter, but this in the average case is so infrequent that after two or three years it may be said to have disappeared. However, this is only partly true of major amputations, for it appears that even after ten or twenty years the natural phantom may still be felt occasionally though never commented on-in contradistinction to painful manifestations which sometimes persist throughout the patient's life.

The average phantom, then, is mild, rapidly subsides, and is soon no longer noticed. If we -may speculate, its existence seems to depend upon some change in the activity of cerebral centres, related to the amputated part, which appears to be modified by two opposing influences, the spontaneous activity of the resting brain and the distracting, swamping effect of extraneous activity and thought. The behaviour of the phantom suggests that the spontaneous activity of these cerebral centres is intensified at the moment of amputation and then after a time gradually subsides, so that one part of the phantom fades out after another. It reappears at longer and longer intervals and the various inducing factors (described later) cease to be effective until at last only the stronger external influences (such as voluntary movements or change of weather) are able to revivify the sensation.

\section{The Impression of Voluntary Movement}

The impression of voluntary movement, the motor counterpart of the sensory tingling, and other features regarded as part of the natural phantom will now be considered.

Most patients are aware of their ability to move at will certain components of the phantom, especially the digits. These willed or voluntary movements are not haphazard, but conform to certain patterns which partly depend upon $(a)$ the level of the amputation, whether distal or proximal, and $(b)$ the degree of sensory awareness of the various joints. These motor phenomena constitute an important part of the phantom, and are complementary to, and in close relationship with, the sensory component already described. Their nature shows that they cannot be purely " imaginary" or psychogenic but depend on physiological processes. Moreover, it does not appear to us probable that they are to be attributed to stimulation of nerve endings in the stump.

These movements do not occur spontaneously but require voluntary effort. Dull and disinterested patients, not having made the attempt, have never experienced them, but when asked to try they usually realize the fingers can be moved, although a few seem unable to initiate the movement. Occasionally, total absence of digit movement seems to be due to functional suppression related to the preamputation condition of the hand (referred to later).

In forearm amputations, the digits can at first be moved in every phantom. Movement is always restricted, often to one-third or less of the full range, and consists simply of flexion and extension, and flexion is easier and persists longer than extension. Wrist movement requires more effort, is not always possible, is of smaller range, and is confined to slight flexion and extension about equally, though extension is sometimes the easier movement.

In arm amputations and shoulder disarticulations digit movement is more difficult, disappears sooner, and is not always possible. Its range is much smaller than in forearm amputations and flexion is again easier than extension, which latter is done with difficulty "against pressure" and is often 
impossible. Elbow movement is rare, and is limited to slight flexion and perhaps a trace of extension.

In the lower limb the same kind of moyements occur as in the upper, but, as the phantom is weaker, they are more difficult to produce, are smaller in range, and disappear sooner. In below-knee amputations, there is no striking difference between flexion and extension of the toes or of the ankle. In thigh amputations toe movement is usually a mere flicker. Movement at the knee was observed on even fewer occasions than at the elbow; it took place always in a flexed phantom and only flexion occurred.

The ability to move the different digits is proportionate to the strength of the sensory tingling. It is freest and persists longest in the thumb, index, and great toe, which can sometimes be moved independently, and it may happen that while all hand digits can be flexed only the thumb can be slightly extended. While movement can still be effected at all joints in the early stages after an arm amputation, the fingers and wrist can usually be flexed simultaneously but not with the elbow; it appears that when attempting elbow movement the patient thinks of the hand and tries to move its position in space, and not of the elbow which he sometimes "cannot even feel." Essentially voluntary but weak movements such as adduction and abduction of the digits never occur; neither does inversion or eversion of the ankle which, as naturally occurring movements in the intact subject, are more postural or associated movements than voluntary acts.

\section{Muscle Actions during Voluntary Movements}

It is interesting to consider the actions of the stump muscles during voluntary movements within the phantom. These are more conveniently studied in the lower limb because of the simpler innervation and movements, and we had more opportunity of observing the effects of denervation. The belowknee amputation may be considered first.

Every willed movement of the toes or ankle is accompanied by a plainly visible partial contraction of the appropriate muscles in the stump. For example, in suitable stumps the superficial calf muscles can be seen to twitch during ankle flexion and the deep muscles during toe flexion. If the extensor group of muscles is denervated by cutting the peroneal nerve the toes can no longer be extended, but flexion is not affected and when the contraction ceases the toes return passively to the resting position. It is also important to note that the position of the toes at rest remains unaltered, an observation suggesting that the resting attitude of the phantom does not depend upon tonic innervation of the stump muscles or on afferent proprioceptive impulses. If, as a further step, the remaining muscles in the stump are denervated all movement is lost. The same phenomenon occurs in forearm amputations ; for example, denervation of the muscles supplied by the median nerve abolishes flexion of the thumb and index, but not of the other fingers.

From these observations it would appear, (a) that the presence of some innervated muscle in the stump is necessary for the performance of willed movements in the phantom, or, put in another way, that the appreciation of willed movements depends on afferent impulses from the muscles which normally move the part; and (b) that the resting attitude assumed by a phantom joint does not depend on impulses from the periphery. The former conclusion is understandable, for it is the normal mechanism of the motor system. It is not enough for the cortex to discharge a motor impulse ; before the phantom toes are felt to move there must be an afferent reply that the muscles have contracted. That this cannot be the whole explanation, however, will appear from the following three observations.

1. Many patients can move the phantom toes but not the ankle although the muscles controlling both joints are present in the stump. This we believe to be due to the relative weakness of the ankle in the sensory phantom, or perhaps, more accurately, to weaker central representation of ankle than of toe movements (even though the muscles concerned are more powerful than the toe muscles).

2. In thigh amputations, in which no toe-moving muscles remain, toe movements are often possible, though small. These toe movements are also associated with a slight twitch in the muscles, which is seen chiefly in the vastus lateralis during extension and the medial hamstrings during flexion, though it is much less than in a below-knee stump. This twitching in the thigh muscles probably represents subsidiary contractions, which occur normally in the more proximal muscles during extensive purposive (digital) movements of the intact limb and have become unmasked by amputation, rather than an acquired secondary motor spread when the principal pathway to the toe muscles no longer exists. Afferent impulses from the slightly contracting muscle fibres in the thigh can evidently be relayed back to the sensory cortex for the toes and the contraction of those fibres is interpreted in reference to toe movement. This, however, happens only in the absence of both the toe-moving muscles and the knee joint, for when a below-knee stump is totally denervated the intact thigh muscles do not 
contract vicariously for the paralysed leg muscles and there is no sensation of toe movement ; it may be that the subsidiary arc is then in conflict with, and suppressed by, stronger afferent impulses from the intact stationary knee joint.

3. There is another important anomaly regarding the muscles. It might be expected that in a proximal amputation the presence of muscles which normally control the elbow or the knee would facilitate willed movements of those joints, but this is not so. Voluntary knee movement, through a limited range of perhaps 20 to 30 degrees, still occurs after, say, six months in only about 5 per cent. of thigh amputations. Our few observations during the first three months or so suggest that knee movement occurs more often at first, though never so frequently as toe movement, and it rapidly disappears. We believe that this paradox is due to the relatively small cortical representation of the knee muscles, which are predominantly postural and are concerned with the least voluntary movements in the limb. Voluntary flexion of the elbow is associated with moderate tonic contraction of the biceps, which is greater than the twitching during the easier digit movement.

The progressive spread of motor impulses from distal to proximal muscles is thus unmasked in amputations, possibly because the proximal muscles are released from their distal attachments, thereby allowing contraction in those muscle fibres which are under the more voluntary control. It is clearly seen after a high arm amputation, when flexion of the digits is associated with twitching in the biceps, especially on its medial side, and extension with a flicker in the lateral part of the triceps; but, if these muscles have been removed in a shoulder disarticulation, twitching then occurs in the posterior fibres of the deltoid.

An interesting observation was made in a man with a high arm amputation who could move the phantom fingers and also, slightly, the elbow. In the course of an ulnar neurectomy for painful neuroma, the elbow flexors were temporarily paralysed by crushing the musculo-cutaneous nerve. This abolished phantom flexion of the elbow, though he could still extend it very slightly and the resting position remained unaltered. Finger movement was unaffected, but the associated muscle twitching was transferred to the posterior fibres of the deltoid; it returned, however, to the medial part of the biceps a few months later after the crushed nerve had regenerated. A slight spread of contraction to the shoulder muscles is often observed during digital movement in the stronger phantoms after arm amputation and the stump sometimes moves slightly.
We have observed a similar phenomenon in quite different circumstances during a local fit due to a cerebral glioma. The movements were mild, were confined to the upper limb, and consisted chiefly of clonic flexion of the fingers, less of the wrist, and very slightly of the elbow, and, in addition, a little twitching was visible in the posterior fibres of the deltoid exactly as happens during voluntary digital movements after high amputation.

This overflow or spread of impulses from, as Hughlings Jackson (1931) put it, the most voluntary to the most automatic muscle groups, that is, to those muscles least used in initiating voluntary movements, is what happens during the more extensive purposive movements of the intact limb, in which the subsidiary proximal muscles are progressively activated in support of the leading part, the digits. The similarity of the phenomena in local epilepsy to those following amputation supports the contention that the latter are intimately associated with the sensori-motor cortex. Further, this relationship between cortex and limb muscles is indicated by the fact that the fibres which contract (for example, in the vastus lateralis) during the movement of phantom digits are those which are recognized as being more directly under cortical control (phasic fibres) than the more postural fibres in the remainder of the muscle (Denny-Brown, 1929).

Having established an association between the impression of voluntary digital movements and twitching of the stump muscles, it is of interest to follow this relationship as the phantom weakens over the course of years. The range of movement and the muscle twitching both decrease gradually with the diminishing strength of the phantom. There is, however, a striking difference in the relationship of these two motor phenomena to their sensory counterpart, depending upon whether the amputation is through the proximal or the distal segment of the limb. It will be described for the upper limb as the stronger phantom sensations are here more easily appreciated.

In a forearm amputation, finger movement is at first easy and there is considerable, though nothing like maximal, contraction of the associated muscles. At this stage digital tingling frequently appears spontaneously and is easily induced by merely thinking about the phantom, without any thought of movement. Later, after a variable period of say six months to two years, when the phantom has weakened so that digital sensation cannot be felt at will and only rarely appears spontaneously, it can still be readily induced momentarily by moving the fingers and there is a slight muscle contraction; there seems to be a tingling sensation of the resting 
phantom in addition to the sensation of movement, but it is difficult to be certain about this. The ability to induce movement persists for a long time, and in the few cases in which it had disappeared by two to three years the muscles had also ceased to contract. These patients often say in the later stages that they " never feel the fingers except when moving them." As the range of movement fades to nothing, so the muscle contraction disappears and the phantom fades out.

In an arm amputation, on the other hand, the muscles have.much less influence in inducing the phantom. In the early months the strength of the digits, and the tendency for them to appear spontaneously is much the same as in forearm cases, but the ease and range of movement and the degree of twitching in the stump muscles is considerably less. As the phantom weakens, voluntary movement rapidly becomes " more difficult," " the fingers are slower and stiffer than they were," and he has "to work harder to move them." We gained the impression from some cases that the muscle contraction increased for a period, probably due to the extra effort required to move the digits and especially the wrist. But a stage is soon reached, at perhaps twelve to eighteen months, when the ability to move the fingers has gone, although the sensory phantom still appears quite plainly, and " the fingers have become locked" or "the fingers seem to be held firmly in a vice" (but without discomfort). In a weakening phantom the increased effort to move the fingers in the later stages often seems to increase the tingling, presumably due to intense concentration. Slight muscular twitching continues, however, often for many months after the cessation of digit movement; it would seem that the afferent impulses from the later twitchings in these subsidiary muscles become too weak to attain the necessary threshold in the related sensory cortex.

In proximal amputations it seems that for a period after the ability to-move the digits has apparently ceased, when " the great toe is very stiff-I don't feel it enough to move it"-it is possible by continued concentration for a minute or two to overcome the stiffness slightly, when "it seems the great toe is loosening up a little" and can be moved slightly. It is likely that the simultaneously increased tingling also results from the concentration-a kind of facilitation.

Voluntary movements thus disappear some time before the sensory tingling (and often before the digits reach the stump) in proximal amputations, but not until a considerable time after it in distal amputations. There is, however, no essential difference in the length of time before the spontaneous tingling disappears. The distal muscles, therefore, have an important influence on the phantom, in that they remain the only means of inducing it for a time after it has faded from consciousness. During this period the phantom must still exist at a subconscious level, otherwise the motor mechanism could not function in relation to it and could not revive it. This ability of willed movements after a distal amputation to induce a sensation of the phantom when it is no longer ' possible by merely thinking about it again suggests that the phenomena of the natural phantom are more closely related to the sensori-motor cortex than to higher centres.

The relationship of willed movements to the sensory side of the phantom is well brought out by the patients' observations when attempting to move each of the three groups of joints at a suitable stage in the life of the phantom. They often say they can move the fingers easily, the wrist with much greater difficulty, and the elbow not at all. When pressed about the inability to move the elbow they reply that they "cannot feel it and don't even know there is an elbow and it is no good trying," or, that they " do not know how to go about it," in spite of having been able to do so at an earlier stage. The freedom of movement, however, depends not only on the sensory awareness of the part, but also (and perhaps chiefly) on the sensory representation (and voluntary control) of the movements concerned ; finger flexion is easier, and has more extensive cortical representation, than extension, even though the muscles effecting both movements are present in the stump.

The fact that the biceps contraction is smaller during digit movements than during the more difficult elbow movements is perhaps evidence of the more extensive central representation of the former. The importance of afferent impulses from contracting muscle on the freedom and range of movement is shown by the greater degree present in distal amputations; but the fact that voluntary movement persists in the digits after it has disappeared at the wrist shows the over-riding importance of central threshold levels.

The last and perhaps the most curious fact is that after the disappearance of willed movements some of the central connexions for innervation of the stump muscles continue to function, for when the patient is asked to "work the muscles" in the stump as much as possible, a maximum contraction is easily produced, but it has no effect in moving the elbow or other joints, nor, indeed, of inducing any sensation of the phantom.

These observations, then, appear to show that: (1) to initiate willed movements in a phantom the motor cortex must first receive guidance from the sensory cortex in which the particular movement is 
still represented consciously or subconsciously; (2) the highest centres can activate stump muscles by two routes: (a) via the sensory cortex, when the muscle contraction and the joint movement in the phantom are never maximal and both gradually decrease and eventually disappear ; $(b)$ by a more direct route without the controlling influence of the sensory cortex, when the contraction is maximal, produces no sensation of the phantom, persists after the phantom has disappeared, and does not diminish with the passage of time ; (3) proprioceptive impulses from muscles do not initiate willed phantom movements, for these are a function of the sensori-motor cortex, but they are essential for the. registration of the impression of such movements ; (4) the phantom shows clearly that proximal and distal muscles are under different nervous control.

\section{Automatic Knee Movements}

An interesting fact concerning knee movement, though not voluntary movement, may be mentioned here. More than 10 per cent. of patients had observed that the phantom knee sometimes moved spontaneously through as much as $\mathbf{9 0}$ degrees, but "it is just automatic." This type of movement, which results in the knee being " sometimes straight and sometimes bent," occurs in various circumstances, such as : (1) during a change of body posture, for example, a knee which is straight when the patient is recumbent may bend to a right angle when he sits. on the side of the bed so that the foot appears to hang down, or when he turns on his side in bed with the normal leg flexed so that " the legs are alike" ; (2) as a visual reflex, for example, when the patient is walking on crutches the knee may automatically flex about 45 degrees if there is an obstruction in the path of the untelescoped phantom foot, or, again, to avoid hitting the steps when climbing stairs, but not when descending. These automatic changes of attitude within the phantom, in which a new position suddenly appears, cannot be voluntarily initiated or prevented, are instantaneous, and the movement is never noticed; attempts to observe the stump muscles during the movement were unsatisfactory. They have no connexion with voluntary knee movements (although the toes can usually be moved) for patients say that " the knee cannot be moved except when it moves itself."

Automatic movements did not occur at the distal joints and only rarely at the elbow and then chiefly when in bed. They evidently reflect the normal, more automatic control of the predominantly postural muscles in the thigh, and may be mediated through reflexes at a lower level in the nervous system than those concerned with the sensory phantom and its voluntary movements.
The change of position which enables the phantom foot to avoid an obstruction can hardly be due, as has been already pointed out, to an inability of the phantom to enter a solid body; were. it so the attitude of the phantom would be constantly changing, either at a joint, which rarely happens, or where the phantom joins the stump, causing it to lose correct alignment, which never happens.

Yet another observation, which seems to concern a mechanism intermediate between voluntary and automatic knee movements, was made by a few patients who noticed that if they "tightened" the knee they could feel the patella tighten and move slightly upwards; this was associated with a prolonged moderately strong tonic contraction of the whole quadriceps, but there was no movement of the phantom knee.

\section{Involuntary Spasmodic Movements}

Involuntary spasmodic movements, which are often painful, will be referred to later. They are regarded as an overlay, functional manifestation, without any relation to sensori-motor cortex.

Factors affecting the Appearance and Strength of the Natural Phantom

1. The Stump.-Pain in the stump merely overshadows the perception of the phantom, and hence during healing may delay its appearance for several weeks, or later, during recurrent inflammation, cause it to disappear temporarily. Attention is also diverted from the phantom by severe pain elsewhere. Mechanical stimulation of the nerves does not affect the intensity of the natural phantom, and trauma has no effect beyond making the stump painful. Contraction of healing muscles causes discomfort and is automatically avoided, so that it is generally a month or two before digit movement is tried and appreciated.

Local conditions in the stump have little other influence, apart, of course, from the possible appearance, after some months, of a superficial sensitive neuroma, stimulation of which causes a pins and needles sensation. A neuroma also leads to the avoidance of voluntary movements when the associated muscular contractions irritate it. On the whole, the length of stump and the method of amputation, whether by guillotine (as in the majority of these cases) or by flaps, made no difference to the phantom, nor, so far as we could tell, did the method of treating the divided nerves. Infection, even when prolonged, had no intensifying action on the phantom. The effect of a persistently unsatisfactory stump in causing dissatisfaction and unpleasant overlay manifestations, and also the rare occurrence of causalgia, are discussed later.

2. Sedatives, Shock, and Toxæmia.-These, by lowering the level of consciousness, delay the appearance of the phantom.

3. Climatic Conditions.-The weather has a marked influence on the vast majority of phantoms, in that they 
are more noticeable in winter, especially during a cold or wet spell. This is the most enduring influence, and it persists long after other inducing and intensifying factors have ceased to be effective. After two or three years the phantom may be noticed only when there is " a change in the weather," or when it is very cold.

4. Thinking of the Phantom.-The patient can at first evoke the phantom, temporarily in abeyance, by merely thinking about it. This voluntary direction of thought is, like other factors, at first uniformly effective but it gradually becomes weaker and finally disappears. It is one of the more enduring influences but it is significant that the patient cannot even imagine he feels the phantom in the later stages, which shows that, like the more obviously refiex factors, it too depends upon, and is influenced by, changes of an " organic" kind.

5. Micturition and other Visceral Reflexes.-These have received passing mention from most writers. There is a brief sensation in the stump or in the phantom during micturition in at least 90 per cent. of thigh and leg amputations alike, during defæcation in about half the cases, and occasionally in association with the passage of flatus. It is usually mild and almost unnoticed ; in fact, more than twenty patients had been seen before it was mentioned casually although, on requestioning, it was found that nearly all had experienced the sensation.

The sensation induced is one of two kinds: (1) a tingling in the phantom and conforming to it as regards position and telescoping; (2) a tingling on the end of the stump, without any reference to the phantom which still retains its normal or partially telescoped position. The sensation sometimes begins by shooting down the stump, perhaps only the last few inches, but occasionally from the groin. The effect is the same with micturition and defæcation, and it appears to remain constant for the individual, as we do not recall a change of response from phantom to stump, although, in this connexion our observations were few.

The tingling has a constant time relationship in the act of micturition. It appears just as the bladder releases and the flow starts. Its duration is variable but is usually short, often only momentary, and almost always fades away before the bladder has half emptied. Distension of the bladder sometimes has a similar effect when the appearance of tingling draws attention to a full bladder, previously quite unnoticed. In such cases its intensity increases until the bladder empties, though there is, in addition, a momentary intensification as the flow starts.

The strength and persistence of the micturition phenomenon varies greatly from case to case. Not uncommonly it is stronger than the spontaneous phantom during the first few weeks and may even be the only manifestation of a phantom, but it rapidly fades and is soon weaker than the spontaneous tingling (which has perhaps become more noticeable). In some it is very weak and fades out within a few weeks, in the majority it is mild and persists for several months or even into the second year, and occasionally it is truly painful, so much so that micturition is dreaded, indicating, we believe, individual variations in the threshold to pain.
A similar sensation commonly accompanies defæcation just as evacuation begins, and, as with micturition, is either in the phantom or in the stump. When both micturition and defæcation responses occur, the former is usually the stronger and more persistent, but either may occur alone. It is difficult to assess the importance of straining. Many say the effect is stronger during constipation, but it seems that the sensation usually appears just for a moment as the sphincter relaxes and does not persist during defæcation, nor is it present during preliminary straining. Nevertheless, it is likely that previous visceral distension increases the response, for the micturition effect is often said to be strongest in the morning, especially if the bladder is distended on wakening, and the defæcation response during periods of constipation.

In upper limb amputations the effects just described. never occurred, but in a few cases, less than 20 per cent., a momentary phantom sensation was induced in other ways, for example : (1) during the act of swallowing very cold water, or more rarely in other individuals, very hot water (retaining it in the mouth had no effect), and occasionally when yawning; (2) washing the same side of the face, neck, axilla or upper chest with cold water ; (3) shaving the same side of the face or neck. Performance of the last two acts was occasionally very unpleasant. The sensation, unlike that in the weaker lower limb phantoms, nearly always begins by running down the stump usually from the shoulder or lower part of the neck, and may, or may not, continue into the phantom.

6. Muscular Action.-The effect of the muscle twitching associated with voluntary movement of the digits in reviving a disappearing phantom has already been described.

7. Loss of Balance.-When the patient stumbles or slips on crutches a strong sensation shoots down the limb, the phantom is felt momentarily, and simultaneously the stump moves out as if to save the patient from falling. Later, the stump may move without any sensation of the phantom. This is one of the more enduring reflexes and generally persists for a year or two. It is interesting to note that the movement of the stump usually changes from putting it out as if to stand on the foot to flexion of the thigh in order to avoid injury to the end of the stump in falling.

8. Instinctive Actions.- It is quite natural that, for a time, the missing part should seek to take its place along with the other limbs in the performance of more or less automatic every-day actions, as in dressing, using the hand to pick up objects or catch a ball, stepping out of bed on the missing foot, crossing the knees with a thigh stump, or, while in bed, moving the stump if anyone is about to sit where the foot appears to be. When the stump begins to move the mistake is realized and simultaneously there is often, but not always, a momer.tary sensation of the phantom. Individuals vary greatly in this tendency to use the missing limb and some assert they never did so. These instinctive actions generally cease after a few such incidents, usually within a few months, for the patients " quickly learn to avoid making mistakes." 
It is interesting to speculate why in these instances the network of central activity concerned in simple, subconscious, and more or less automatic actions involving centres for several limbs should evoke a conscious sensation in the sensory cortex related to the amputated limb and not elsewhere. Is this cortex in a hypersensitive state, enabling the normally subconscious reflexes locally to reach a conscious level ?

The sensations during instinctive actions are weaker and disappear sooner than those associated with the fright of falling, as would be expected, for the intensity of reflex action may be presumed to be considerably less. The reason why mistakes soon cease to be made may depend upon re-education, as these reflexes are initiated centrally and presumably at a higher level than those in response to fright, and overbalancing, which - are induced by peripheral stimuli and are therefore more automatic.

9. Vision.-Vision sometimes provides an effective stimulus, for example, seeing a man with an amputation may cause the phantom to appear. The occasional adoption by the phantom of the position in which the limb had been splinted before amputation (described later) probably depends on vision more than other sensory impressions. Also, it seems to provide the stimulus for some automatic knee movements. The chief importance of vision, however, is its effect in modifying the natural phantom by suppressing part of it or by adding sensations, for example, superimposing the visual impression of the original wound (described later). There is no evidence that vision is fundamentally concerned in the genesis of the phantom phenomenon, which appears in similar form in men who have been blind from the time of wounding.

10. Fright.-A sudden fright, for example, from a loud noise, is sometimes associated with a momentary sensation of the phantom. Fright probably plays a part in the reaction to falling.

11. Emotion.-Emotion such as anger or excitement makes the patient forget the phantom.

12. Learning to Write.-This never induced the phantom, except in two strongly left-handed men with left-arm amputations.

13. Wearing an Artificial Limb. - Wearing and walking on an artificial limb has a striking effect. Nearly a hundred men were fitted with temporary, above- or below-knee, wood-plaster limbs nine to eighteen months after amputation, which enabled them to get about again without crutches and usually without sticks. Almost without exception the phantom was never felt while wearing the prothese but often reappeared when it was removed. This effect may be partly psychological due to greater freedom of locomotion, but it is likely that other factors are important. It was, for instance, sometimes observed that, when the patient was standing, if all the body weight was transferred to the normal leg, and the stump was slightly lifted out of the socket, the phantom appeared, but it disappeared when the patient was standing equally on both legs. It was not noticed when walking. Similar observations suggest that in the upright posture correct distribution of body weight, balance, and the automatic actions in walking may have an important inhibitory effect. We had no experience of artificial arms.

14. Time of Amputation.-There was no noticeable difference in phantoms following amputations done at the time of wounding, or within a few weeks or months. Phantoms did not appear, however, in several cases of late thigh amputation, done eighteen months to two years after wounding, with co-existing complete sciatic nerve lesions in which the total loss of sensation in the anterior half of the foot was verified before operation. As the usual lower-limb phantoms have often disappeared by eighteen months, the question arises whether the nonappearance of a phantom in such cases can be attributed to the fact that the cortex had already long been deprived of its normal sensory connexions in the sciatic territory, especially the forefoot ; in other words, whether denervation has an effect similar to amputation on the cerebral cortex, except that after peripheral nerve injuries the presence of the foot in some way inhibits the appearance of a phantom.

\section{Relation of the Phantom to Solid Structures}

One of the most striking and curious features of a limb phantom is its ability to pass through solid structures unimpeded and without arousing anyo ? feeling of obstruction or unpleasant sensation. It $\$_{\infty}^{\infty}$ behaviour in relation to solid structures may bet 응 considered under three headings :

1. The relation of the whole phantom to the patient's body. The phantom always moves in alignment with the stump, so that a hand which is $\vec{\omega}$ normally in front of the trunk can be made, by moving an arm stump backwards, to pass straight through the patient's body.

2. Its relation to external objects. Again, the phantom maintains its shape and its relation to the stump, and ignores the presence of an obstruction, so that it goes through, for example, a wall or a table. When in bed the foot of a right-angle phantom appears below the mattress, but if the knee is only slightly flexed the foot is felt to be inside the mattress.

An interesting phenomenon was met with in several cases, in all of which the fingers were fully telescoped and projected downwards from the end of an arm stump. When the stump was slowly approximated to a wall, the fingers seemed to enter it (the wall) and nothing happened until the stump touched the wall when they were instantly displaced either sideways or inside the lower end of the stump, as if they had been pushed backwards. It made no difference whether or not the patient was watching and there was no associated pain or other sensation. When the stump was removed the fingers instantly returned to their former position. This effect was 
not observed when telescoping was incomplete nor in the lower limb, possibly because the toes are weak and have often disappeared before reaching the stump.

The only change that occurs, then, in these two groups is when strong phantom digits, attached to the stump, appear to occupy a space which, according to the normal sensory impulses from the end of the stump, does not exist. In this conflict the normal cutaneous sensory information prevails and the fingers are displaced into the stump without any sense of resistance, as sometimes occurs spontaneously in the natural course of the phantom. It is strange that, apart from this special circumstance, the phantom, which seems to maintain such perfect connexion and harmony with the stump, can yet be so detached from the patient and from external reality. The fact that it ignores other (normal) impressions, bodily or visual, may be due, in part, to weakened central neuronal connexions representing the gaps in the untelescoped phantom, and possibly in part to the active digital cortex taking precedence over other (for example, visual) impressions. The only exception is when the fully telescoped fingers become so to speak part of the stump and the normal cutaneous impulses are in direct (anatomical) conflict with the abnormal impression of the digits which have to give way. But most interesting is the ability of the normal stump to engulf the phantom digits, temporarily or permanently.

3. The relation between different parts within the phantom. Occasionally the attitude of the hand is such that one part feels to be firmly in contact with another and may dig into it, for example, the tip of the adducted semiflexed thumb pressing against and piercing the lateral side of the semiflexed index ; or, the palm is felt because the nails of the fully flexed fingers are digging into it ; or, in the mildest cases, there is merely a sensation of light contact between the tips of the semiflexed, opposed index and thumb. Such impressions of contact, and even penetration, are often uncomfortable and have a burning and sometimes very painful quality. They can occur when all the muscles directly concerned are missing, as in arm amputations, and it is difficult to see how irritation of divided afferent nerve fibres from those muscles or of pain fibres could arouse such complex sensations. This is the only circumstance in which a part of the phantom feels to make any contact, namely, with another part of itself, and in which there is an accompaniment of pain which is more severe when the pressure is unusual. These considerations suggest that the sensations probably depend on psychogenic factors.
Increased Tone in the Muscles controlling the Stump

It is interesting to note that in most cases there is an increase of tone in the muscles moving the stump during the induction of general anæsthesia. This excessive tone affects principally the flexors and is manifested either in spontaneous movements of the stump alone or by its exaggerated movement when all the limbs move. It can be demonstrated, in the absence of spontaneous movement, by passively abducting an arm stump which induces clonus. This movement during operations on the stump, when the patient is not fully anæsthetised, is annoying to the surgeon, and it was this that first drew our attention to the phenomenon.

The reason for the increased tone in the muscles controlling the stump, which is in excess of that occurring simultaneously in the normal limbs, is not clear, but two possibilities suggest themselves. It may be an expression of heightened activity in the related centres (for the muscles moving the stump), associated with that in the adjacent cortex representing the amputated part; or, it may be a release phenomenon, distinct from that which removes higher control during induction of anæsthesia. Is it possible that the tone in proximal (supporting) muscles is normally under some inhibitory control from the distal (leading) part of the limb? In other words, does a mild release phenomenon with increased tone result from cutting peripheral nerves distal to the muscles (though less than that after isolating them from cerebral control in upper motor neurone lesions)? If so, this might be regarded as the motor counterpart of the sensory phenomenon which expresses itself in tingling.

It is probable that this increased tone is related to the well known spontaneous jumping of the stump during the early weeks after amputation. It may be that these occasional jerkings of the stump, often amounting to clonus, are sometimes initiated by mechanical irritation of the divided nerves, but a similar quivering occurs reflexly in response to other stimuli such as a fright or during a yawn or a stretch; also, it frequently happens in the absence of any obvious reflex cause as when the patient is lying quietly in bed especially just before or after falling asleep. There is considerable individual variation in the severity and duration of this jumping, but it gradually subsides and usually disappears within a few months ; in the early weeks it is sometimes associated with a momentary sharp or cramp-like pain in the stump muscles but without a phantom sensation. We regard it as a motor manifestation of the natural phantom which 
gradually weakens just as the sensory counterpart, though it often continues to be revealed during induction of anæsthesia.

\section{Excessive Sweating in the Axilla}

This occurs after some forearm and most arm amputations, and without any painful or causalgic manifestations. It gradually decreases but may continue for two or three years, and it is sometimes so marked that perspiration is continually running down the chest wall, occasionally even in cold weather and although the other axilla is dry. It is more frequent and more marked the more proximal the amputation. It is regarded as an expression of heightened cerebral activity affecting the sympathetic system and comparable to phantom tingling and stump clonus.

So far only manifestations which are regarded as part of or associated with the "natural phantom" have been described, but others which may be superimposed remain to be considered. Such additional features are present in only a minority of cases, are peculiar to the individual patient, and do not constitute a fundamental part of the phantom. They are, however, sometimes unduly conspicuous and troublesome, in consequence of which, from the standpoint of phantom physiology, undue attention has been given to them in the past, and there has thus been a tendency to think of phantoms generally in terms of these uncommon manifestations. Reference to a disproportionate number of these patients to illustrate the different effects gives a false impression of their frequency, but not, of course, of their importance, for therapeutically these are the only features that matter. The effects to be described come from two entirely different sources, one from stimulation of nerve ends in the stumpthe most simple organic manifestation-and the other of psychogenic origin depending on the highest cerebral levels-'the most complex phenomenon.

\section{THE NEUROMA SENSATION}

It has long been surmized that the various sensations which constitute the phantom are closely connected with afferent impulses originating in the stump. We believe that the only sensation (apart from the impression of voluntary movement) which can with certainty have such a peripheral origin is that resulting from mechanical stimulation of a neuroma. It may be, however, that the occasional spontaneous, momentary sensations, usually mild but sometimes stronger and even knife-like, and localized to small areas in the phantom, are due to irritation of individual fibres in the divided nerve; they are most noticeable during the first month or two after amputation and then gradually disappear.

Although a neuroma forms on every divided nerve, only those situated superficially are liable to repeated trauma. Percussion produces an instantaneous sensation of "pins and needles" which is referred to the cutaneous distribution of the nerve but is always easily differentiated from the tingling of the natural phantom, for it is much stronger, is only momentary, and never occurs apart from mechanical irritation. The distinction is well brought out by stimulating an ulnar neuroma, when the pins and needles in the little finger is in sharp contrast to the tingling in the phantom index and thumb; but even with a median neuroma the patient has no difficulty in distinguishing the two sensations. Further, stimulation of a neuroma has no effect in intensifying or inducing the phantom, which is, indeed, unaffected by troublesome neuromas. Excision of the neuroma, or division of the nerve, abolishes the sensation, but the phantom tingling is unaffected. It seems, therefore, that impulses from the exposed axons, at least those resulting from mechanical stimulation, have no part in the genesis of the natural, spontaneouslyappearing phantom. Furthermore, the neuroma, which takes several months to form, cannot be responsible for the phantom which appears immediately after amputation.

It must, however, be emphasized that the sensations of the natural phantom and of the neuroma response, though quite distinct in character and presumably also in origin, have an important mutual relationship in that the latter is always located correctly within the phantom as regards position and degree of telescoping, even when the fingers have retracted within the stump. There must, therefore, be some integration at a high level in the nervous system. After the digits have lost their definition, the neuroma response is referred to the skin on the end of the stump, where it produces simply a pins-and-needles or weaker tingling sensation without reference to digits, and this persists after the phantom has disappeared.

The sensation, strong at first, gradually weakens, but the effect of stimulating the main nerve trunks to the digits never disappears even after the phantom has faded out; on the other hand, the smaller cutaneous nerves, such as the external cutaneous or the branches of the femoral, produce a much weaker response which is at first referred correctly to the phantom, but weakens rapidly as the phantom shortens, and, after being referred to the stump for a short period, disappears even before telescoping is complete. Thus, the sensations from even the poorly represented long segments of the limb are 
referred to the normal skin on the stump for a short time before they disappear. Moreover, sensation induced by stimulating nerve ends of greater and of lesser sensory importance when the phantom is partly telescoped may at the same time be referred to the phantom and to the stump respectively. This difference of effect is presumed to depend upon a gradual change (? lowering) in the level of cortical activity affecting unequally the strongly and poorly represented parts.

In some amputations, mostly of the arm, the neuroma is unusually sensitive and produces an excessively strong pins-and-needles sensation, sometimes referred to as pain, which extends proximally up the stump. This recalls $(a)$ the inclusion of phantom digits within the stump, and $(b)$ the passage of sensations, induced by certain reflexes previously described, from shoulder or groin down the stump before entering the phantom (micturition effect). It may be that the three phenomena are due to the same central mechanism.

The neuroma response is the same sensation that underlies Tinel's sign, but in amputations the peripheral reference is modified by telescoping, which never occurs in peripheral nerve injuries.

\section{MODIFICATION OF THE PHANTOM FROM A HIGHER CEREBRAL LEVEL}

The phantom is sometimes modified in such a manner that it is reasonable to assume it is dominated by a higher level than the sensori-motor cortex. In the majority of such cases the natural part of the phantom is unaffected and there are mild superadded sensations which derive chiefly from the memory of previous experiences. In others, the additional sensations are stronger and perhaps painful, or the underlying phantom is itself modified, either by suppression or by distortion into unnatural forms. In the rare, most severe, cases the superimposed sensations may be extremely unpleasant and painful, dominating the phantom and causing the patient to complain bitterly about " the nerves."

We are well aware that we are here about to embark on a highly controversial aspect of the physiology of phantoms, and that many of the phenomena now to be considered have by many been regarded as arising from purely peripheral nerve irritation. Whilst we in no way wish to deny the role of peripheral nerve ending in the genesis of certain types of pain in stump and phantom, we feel, nevertheless, that the probability of a central origin for some at least of these superadded sensations has never received adequate consideration.

\section{Superadded Sensations of Pre-amputation Impressions}

The majority of these sensations can be traced to impressions received originally via the sensory system, though in some instances vision may have participated. They are, therefore, a recrudescence of pre-amputation experiences peculiar to the patient and must be distinguished from the basic tingling that is common to all. Brief consideration of their qualities and the factors associated with their development brings out some interesting and suggestive facts.

The sensations may be divided into three groups, depending on the relationship of the parent sensation to the time of wounding : (1) the revivification of a sensation experienced in the limb before wounding, sometimes even several years previously and apparently forgotten, for example, the discomfort of an ingrowing toe-nail or a corn, compression of the toes in a tight boot, the impression of a split finger nail, a painful whitlow, the sensation of a finger ring ; (2) the wound itself ; (3) the persistence of a sensation experienced between the times of wounding and amputation, a period often of several weeks or months duration, for example, the pain of suppurative arthritis, the sensation of a traction pin, pain in relation to pressure points and splints, the sensation of lice crawling under a plaster.

We believe that all these sensations, to which the adjective " superadded" is applied, show features which distinguish them from the pure phantom. They are noticed only occasionally and are mild and transient, usually only momentary in comparison to the more persistent awareness of the natural phantom, and they disappear early. They often appear in the absence of phantom tingling, for example, the ingrowing toe-nail sensation may be present without toe tingling, or a wound sensation be isolated in the gap representing the forearm. Their position in the phantom, however, is always consistent with its shape and telescopic shortening.

A fact of considerable interest, and one that appears to be at variance with the views of many, is that their occurrence had no relation to the intensity of the pre-amputation sensation. For example, in several cases of delayed thigh amputation a slight momentary pain felt in the knee at the time of wounding appeared in the phantom, whereas the later, much more severe and persistent pain of suppurative arthritis in the knee did not. Analysis of about ninety thigh amputations, of which some thirty Pollowed acute arthritis of the knee, often after many weeks of severe continuous pain, showed that it appeared in only a minority of the phantoms, and then usually when the patient had some cause 
for dissatisfaction. It is, in fact, our strong impression that a man who has had much pain and who is glad to have the leg off has no pain in the phantom.

A somewhat related fact is that wound phantoms were more common in the lower limb, although the phantom tingling was more vivid in the upper. This apparent anomaly is perhaps due to the more immediately disabling effect of a lower limb wound and the resulting greater mental impression. In the few upper-limb phantoms in which a sensation of the wound appeared, the patients invariably gave a strikingly graphic description of its appearance with details of blood running down the arm, etc., whereas in the lower limb the sensation was simply that of the pain experienced at and shortly after the time of wounding.

The frequency with which a psychologically important sensation persisted in the phantom was quite impressive, and the following example illustrates clearly this association. A soldier in jumping from a truck sprained his ankle. This caused him to fall behind his companions. A few minutes later he was wounded in the same leg just above the ankle, and the pain of the sprain was replaced by a different pain in the wound. He was taken prisoner and a few days later the leg was amputated. He had a typical mild phantom but he noticed principally the pain on the outer aspect of the ankle similar to that caused by the sprain. In the course of the examination he remarked significantly that he would have succeeded in escaping had it not been for the sprained ankle.

These facts suggest that the genesis of this class of superadded sensation is the effect of a psychologically important pain and is not related to the severity and duration of the pain. The slight pain at the time of wounding which is the primary cause of the soldier's troubles, and, in our cases, of his becoming a prisoner of war, is more apt to appear in the phantom than the later severe pain of suppurative arthritis, which is merely an incident in treatment. In other words, the appearance of such superadded sensations probably depends largely on psychological factors.

\section{The Possible Modifying Influence of Pre-amputation Posture}

The reason why the elbow or knee is straight in some cases and flexed in others is not clear. After some delayed amputations the attitude of these joints reproduced the position of the previousiy splinted limb-the patient usually volunteered this information. This was seen most clearly in a small group with the knee slightly flexed at about 135 degrees, almost all of whom had been splinted on a Braun's frame for several weeks or months ; or, again, in cases with a fully flexed elbow after the limb had been splinted in that position. Occasionally it corresponded to the position of the limb when wounded ; for example, a truck driver with a slightly flexed phantom elbow had been holding the gear lever. The rare cases with full flexion of the knee are harder to explain, though some of the patients, for example miners, said they had normally worked in that attitude. There can be little doubt that some impressive pre-amputation position, especially when acute or obtuse-angled, can modify the attitude of the phantom.

In the large majority of cases these joints adopt an attitude either of full extension or of right-angle flexion. Statistically, there is no apparent relationship to the previous position of the splinted limb, or even to the patient's recollection of the injured limb when wounded or, later, on the stretcher before an immediate amputation ; it was the same in some but not in others. Further, it must be uncommon for a lower limb to be splinted with the knee at at right angle, and some patients were unconscious or too shocked to appreciate its position. In then majority of phantoms, the knee was fully extended and the elbow was flexed at a right angle. This isothe attitude characteristically adopted in hemiplegia ${ }_{\circ}$ and we are tempted to see in it the reflection of a postural pattern in the central nervous system $\doteq \overrightarrow{0}$ Denervation of one or both groups of stump muscles $\frac{0}{\infty}$ had no effect on the attitude of the resting joint, which seems to indicate a fundamental cerebral control of the position of joints. If, as we suppose, the relative complexity of voluntary movements is proportionate to the degree of their cortical control, may not the attitude of proximal joints be assumed to represent a lower level central control of postural tone (just as automatic knee movements are supposed to reflect low level reflex movements)? But this leaves unexplained why some elbows are extended and some knees are flexed, without even any apparent relationship to previous posture.

We do not agree with the view that it is the rule for pre-amputation pain and posture to be "frozen " or perpetuated in the phantom. When an unusual sensation appears in a phantom it represents a previous psychologically important experience more often than an immediate pre-amputation "frozen" sensation.

\section{Suppression of Part of the Natural Phantom}

This results chiefly from previous visual impressions and affects either the sensory or the motor component. 
For example, an airman, severely wounded in the foot, had the leg amputated a day or two later. The phantom was quite typical except that he had never felt the first two toes nor the medial border of the foot, which is normally the stronger side, but he could feel the lateral part of the foot and toes easily. The explanation of this curious distribution appeared when he remarked that he had seen the foot before amputation and remembered clearly that its medial half " had been shot away leaving a gap."

Another example is that of a soldier whose second toe was removed together with the distal part of the metatarsal a few days after being wounded; this left a "V-shaped gap" which he had seen during dressings. Several weeks later it was necessary to amputate through the leg. The usual phantom appeared in which he could feel all the toes except the second and the V-shaped gap which was " missing." (He could still feel the phantom eighteen months later when the posterior tibial nerve was divided for a painful neuroma; the whole phantom unexpectedly disappeared completely immediately after this peripheral operation and had not re-appeared during the following twelve months-this case is referred to again in connexion with operations.)

Not only may the tingling of the natural phantom be completely suppressed in a defined area in accordance with a psychologically-important pre-amputation impression, without affecting the remainder of the sensory phantom, but, on the motor side, voluntary movements may also be suppressed locally.

For example, a man with a forearm amputation had an ordinary phantom in which he could feel all the digits and could voluntarily move all except the ring finger, which remained rigid in its slightly flexed attitude, although the stump muscles twitched as usual. The explanation became clear when he remarked casually that the finger had been stiff since an accident several years previously.

A further example is that of a man, seen two months after an arm amputation, in whom the fingers were in the semi-flexed clawed position that they were in when he was wounded (he was holding on to a gun rail). They could not be moved, whereas he could move the wrist slightly and, with greater difficulty, the elbow. Attempted finger movement caused a cramp in the stump muscles, but there was an associated twitching in the muscles just as during movement of the wrist and, in greater degree, of the elbow. The motor phenomena were thus normal apart from the suppression of afferent impulses from the contracting muscles, over which the impression of tightly grasping the gun rail prevailed.

This phenomenon of suppression occasionally affects the whole phantom, as when it totally disappears following an operation on the stump (referred to later). It may also be the explanation of the rare non-appearance of a phantom after amputation.

\section{The Occurrence of Unnatural Attitudes or Abnormally Free Movements}

Unnatural attitudes occurred in only a few instances, almost always in the hand. The reason sometimes came out in the history, for example, an airman with a forearm amputation had a typical phantom except that the thumb was markedly extended ; the last thing he remembers before being wounded was pressing hard with the thumb against part of the gun in an effort to get it working-the phantom thumb appeared in the same position and was at times slightly painful.

Frequently, however, there is no discoverable reason for the unusual attitude, which is usually a simple modification of the natural position and within anatomical limits, though sometimes rather forced, and they do not worry the patient to any extent, for example, when the fingers are heaped up consecutively on the index as children do, As in the case just quoted they are akin to the superadded sensations in the first group, and seem to depend upon the memory of a previous experience. In rare instances the positions are so unnatural and fantastic, as when the fingers are grossly twisted and interwoven, that they can hardly be related either to any previous experience, or to afferent impulses from the divided nerves and muscles in the stump.

Similar inexplicable behaviour was also seen a few times on the motor side. Normally, voluntary digital movements depending on the usual organic reflexes are restricted in range, consist simply of flexion-extension, and are always associated with slight contraction in the appropriate stump muscles. As an example of abnormally-free " non-organic" movements, a man with a leg amputation always asserted that he could move the phantom foot through the full range in all directions, including inversion and eversion (which we have never found to occur in a natural phantom), and that ankle movement was as easy as toe movement and just as in the normal foot, although there was no sign of concentration or effort such as is often noticeable in the facial expression. The suspicion that this was a purely psychogenically-determined phenomenon was, we considered, confirmed by the fact that the stump muscles remained motionless.

\section{Submergence of the Natural Phantom by an Overlay of Disagreeable Manifestations}

It is these cases which show the most severe disturbances but they are rare-less than 5 per cent. The usual sensory and sometimes also motor manifestations of the natural phantom are obscured in varying degree, and may be completely submerged 
by an overlay of disagreeable painful sensations and gross, bizarre digital distortions beyond anatomical limits. This group includes the spasmodic, involuntary, uncontrollable, and often painful movements of the digits, which are more common after proximal amputations, occur in the absence of voluntary movements, and may disappear after a satisfactory re-amputation ; the occasional irregular jerkings of the stump, usually at times of emotional upset ; and sometimes, we believe, the cramp-like muscular pains in the phantom. These various manifestations are, we would suggest, psychogenically determined and, in a few patients, this suspicion was confirmed by the later development of outspoken functional symptoms.

The painful sensations often have a burning or boring quality, like raw flesh or red-hot needles or even slimy and wet, sometimes limited to the hand, but usually involving the whole limb without any gaps, so that there is no distinction between parts having different degrees of central representation. The pain may be intermittent but, like many psychogenic pains, is often constant and without variation either from time to time, or between different parts of the phantom. The attitude of the proximal segments is hardly ever affected, but in one exceptional case a complete phantom occupied an unnatural position in a straight line obliquely across the front of the body at an angle of 45 degrees to the line of the arm stump; it was continually painful, and, in addition, there were sometimes painful involuntary movements of the fingers, which, however, could not be moved voluntarily.

We had two or three cases showing constant burning pain in upper limb phantoms and a tender hyperæsthetic stump-a condition resembling if not identical with causalgia.

It appears that psychogenically imposed sensations are predominantly painful and never consist of the simple tingling of the natural phantom. - It is also interesting to note that the sensations which may appear in a phantom seem to reflect, as regards their duration, three levels of sensory activity, namely, the psychogenic pain which is constant, the sensori-motor tingling intermittent and of intermediate duration, and the stump neuroma response which is momentary.

From a study of the foregoing groups it would seem that the mind can subconsciously impose in varying degree a great variety of sensations on to the stereotyped natural phantom. The milder manifestations represent incidents of varying psychological importance in the patient's past experience, but their appearance and severity depend upon the personality of the patient. The most extreme development is, we believe, evidence of a hysterical personality. The disturbing mental effect of uncertainty regarding pension, etc., may undoubtedly focus attention on the amputation, and we suspect that this factor is often important in civilian accident compensation cases. Assuming that psychogenic suppression of the natural phantom or imposition of overlay sensations may occur, we often wondered whether the reverse mechanism occurs and if the occasional phantoms in which there was no evidence of fading out were, in fact, for some reason being psychogenically perpetuated. One man, for example, with an unusually persistent and strong phantom had been told by the surgeon that he would feel the nerves for four years and it looked as if he would. What appeared to be a perpetuating influence, dependent on dissatisfaction, was seen in several cases requiring re-amputation, as described below.

\section{SECONDARY STUMP OPERATIONS}

In many cases secondary operations were carried out on the stump-about twenty re-amputations, more thán thirty neurectomies, and many sequestrectomies-mostly from about six months to two years after amputation. Some of these men andळ many others had already had secondary suture of guillotine stumps, re-amputation, sequestrectomy $?$ etc., during the earlier months, often before we saw them, without any noticeable effect on the phantom as far as could be judged from their descriptions $\frac{0}{\sigma}$ Observations before and after our own operations confirmed the impression that operation on the stump, whether or not the nerves are re-divided, had no effect on the phantom except in the special circumstances described below.

Re-amputations were done, (a) to convert an unhealed guillotine stump into a soundly healed one, usually by removing about two inches of bone and suture of flaps, and $(b)$ in a few cases to remove a troublesome, useless knee or elbow. Neurectomy was performed to stop troublesome pins and needles from a superficial neuroma, usually in arm or below-knee stumps. When the nerve contained no muscular branches it was divided high up where the fresh neuroma would not be subjected to future trauma, for example, the ulnar and median nerves in an arm stump were divided in the axilla. Several inches of nerve were excised and the neuroma not removed unless the stump was being trimmed.

Neither operation, generally speaking, had any effect on the strength, telescoping or willed movements of the natural phantom. Occasionally, however, either procedure caused complete disappearance of the phantom. This seemed to happen 
when the patient was particularly pleased with the result of the operation, and especially when the stump had remained troublesome after several previously unsuccessful operations, troublesome not so much from pain as from the annoyance of requiring constant dressing for one or two years, and perhaps also from recurring inflammation. The satisfaction of at last having a well healed stump and discarding the dressing seemed to suppress everything connected with the amputation, including the phantom. One man, for example, had had two unsuccessful operations in an attempt to heal a discharging stump between nine and eighteen months after amputation; the third secondary operation was successful and the phantom, previously quite strong and painful, completely disappeared. He said that he had been very disappointed with the second attempt, after which he began to experience pain in the phantom. This late appearance of pain was observed in several others when they began to realize that the stump was slow in healing and was preventing them getting an artificial leg. It was not seen in the first few months when unhealed stumps were the rule and accepted as a normal occurrence.

Suppression of the phantom was also seen after several neurectomies. The reason for this was not so apparent as after re-amputation, since the operation was done simply to abolish the pins-andneedles from a neuroma. There was, however, usually other evidence in the phantom of functional overlay or suppression, and the patient's mind, possibly unusually suggestible, was evidently ready to assume that the operation would cure "the nerves" completely. This abolition was observed for at least a year : whether it remained permanent thereafter is not known. It is important to appreciate that suppression of the phantom, even when painful, may occur after simple operations on the stump in certain cases, as well, apparently, as after tractotomy and cortical excision, but of these latter operations we have no personal experience.

The disappearance of a functional overlay sensation with the subsequent emergence of the already weakened natural phantom following a satisfactory operation is illustrated by the following case.

A man with a mid-arm stump was first seen two years after amputation. Cosmetically, the stump was bad, infection was present and there was a large redundant fold of soft tissue hanging loosely from the end. $\mathrm{He}$ experienced a constant unpleasant dragging sensation and feeling of weight on the end of the stump, which he said had always been present, and he could recall no other sensation. The stump was repaired by removing one or two inches of bone, and the flaps healed immediately. He was delighted with the new stump and " glad to be rid of the bandage." He then remarked that the unpleasant dragging sensation had disappeared, but, instead, there was a vague sensation of the index and thumb on the end of the stump which he had never felt before; it was a mild tingling, quite pleasant and " not disagreeable like the dragging." This was evidently the natural phantom and it was just at the expected stage for that period after amputation. It is assumed that the gradual fading of the natural phantom had been taking its usual course and it entered consciousness only after the functional overlay sensation had disappeared.

\section{DOUBLE AMPUTATIONS}

Our observations on double amputations are few and incomplete. In general, the various features of double phantoms are about the same on both sides. There are, however, usually minor differ ences of detail. The two phantoms may appear simultaneously or independently indicating, presumably, that their respective cerebral connexions function separately. Again, one phantom may disappear before the other; for example, we observed over a long period a man with arm and thigh amputations on the same side; the phantoms were at first typical though the hand was always stronger and was still readily perceived after the foot had disappeared at about eighteen months. It was our impression that the phantoms after symmetrical amputation were, on the whole, weaker than after the removal of one limb. Hemispheral dominance had no noticeable influence on double, or, seemingly, on single phantoms.

During the Ardennes fighting in the winter 1944-5, we saw six men with severely frostbitten feet necessitating double amputation, usually below the knee, after six or eight weeks. The amputations were done separately with an interval of ten to fourteen days, which afforded a good opportunity to get the patients to compare the sensation of the phantom foot with the dead foot. These observations supported previous conclusions about phantoms generally. The anterior halves of the feet and usually a part of the heel were black and hard frem dry gangrene. The pre-amputation sensations consisted of momentary sharp pains, often caused by movement, in the demarcation zone across the foot, and, sometimes, constant pain in the heel due to pressure. The pain occasionally radiated into the toes but never appeared there alone, and there was no phantom-like tingling. It was usually intermittent and relatively mild, and unlikely, therefore, to overshadow any tingling in the dead toes had it been present.

After the first amputation, typical phantom tingling appeared in the toes, especially the great 
toe, usually during quiet periods, and it remained for at least several minutes. When the tingling was stronger it also appeared in relation to the heel, instep, etc. In addition, the momentary preamputation pains in the foot were sometimes superadded, but they disappeared within two or three weeks, leaving the patient more conscious of the phantom toes. Tingling also appeared if only the toe was removed by completing the line of demarcation at its base, which meant dividing only the last bit of dead joint-capsule and flexor tendon. The difference between the pains in the gangrenous foot and the tingling in the phantom toes was easily appreciated ; they appeared at different times, the one in response to movement and the other when quiet. After the second amputation, the two phantoms appeared either together or separately, and they were not necessarily identical in strength (the second was the stronger and sometimes the only. one to appear) or in distribution (depending on pre-amputation sensations).

As regards the micturition effect, there was no definite instance of it occurring before amputation, but it always appeared afterwards, usually as a tingling on the end of the stump, which was sometimes preceded by a shivering sensation which ran down from the thigh. After the second amputation the sensation always occurred simultaneously in both legs and was usually similar in strength, though sometimes it was stronger on the more recently amputated side ; its strength, therefore, seemed to depend on the responsiveness of the related central connexions, as the stimulus from the bladder was the same for both. During the first few weeks, the micturition effect was often stronger than the spontaneous phantom and was sometimes the only sensation to appear, but it rapidly faded and soon was hardly noticeable.

Voluntary movement of the phantom toes was not possible for a month or two and at first was often unilateral, appearing first in the earlier amputated leg, when toe movement induced tingling on that side but not the other.

Multiple phantoms can thus appear independently or, in certain circumstances, simultaneously though modified by local features such as the age of the phantom. Further study of double and especially asymmetrical amputations might yield much interesting information.

\section{MINOR AMPUTATIONS}

Partial removal of a hand or foot or even part of a digit, is followed by the appearance of a phantom which is essentially the same as after major amputations. It is, however, less noticeable, possibly because the absence of a digit causes little inconvenience, and mild tingling in a finger when the rest of the hand is normal is not so strange as when the whole arm is missing. Nevertheless, digital tingling is not much weaker after simple removal of a digit than after amputation of the whole limb, and it has the same quality. Any slight difference in strength is thus disproportionate to the size of the missing parts. In general, the strength of any phantom may be said to depend on the vividness of its terminal phalanges ; it does not seem to matter how much more of the limb is removed, for that often results merely in the appearance of additional, and usually weaker, parts which, as it were, support the digits.

When several fingers are removed individually, there is the same difference in the strength and persistence of their several phantoms as after a major amputation, so that all may be felt during the first year or two, but the thumb and index are stronger than the others (the latter appear only during the winter), and they persist for a year or two longer. The toes are considerably weaker; the great toe rarely lasts longer than a year or two and the lesser toes may never appear.

The three main features of major phantoms ma also occur after minor ones, namely, the spontaneou natural tingling, the pins and needles from a digitaf? neuroma, and, very rarely, constant pain or other disagreeable features, probably of psychogenio origin. Minor phantoms are, however, generall $\vec{x}$ mild and rarely troublesome. Phantoms afters $\stackrel{\infty}{\infty}$ minor amputations, then, are minor only in the sense that a small part has been removed and not as regards their strength, in which respect they are similar to larger phantoms.

\section{DISCUSSION}

This study of limb phantoms has shown that a phantom is a natural and almost constant sequel to amputation, and not, in fact, a pathological manifestation. Fundamentally, it is a stereotyped though complex phenomenon. Occasionally, ad- 훙 ditional features introduce such marked variations that before seeking a general comprehensive $ᄋ$ explanation for the phantom it is advisable to $₹$ analyse the various sensations which may occur 의 and the conditions under which they appear. The $D$ more general, almost universal, properties may be considered first, and then the epiphenomena which $\bar{N}$ are peculiar to the individual.

We found the great majority of phantoms were $N$ painless. When, for instance, ninety-eight men in N one batch were questioned at periods up to six 
months after amputation in 1940, only four complained of "the nerves." This incidence of pain is much lower than is often supposed and, indeed, our series gives a different general impression of phantoms from that described by other writers due, perhaps, to the distinctive type and age-group of our patients. Lhermitte (1939) found only three instances of painful phantom in his thirty cases, but implies that this is much lower than the commonly supposed incidence, whereas Riddoch (1941) states that nearly half are painful. However, it may be that pain is more common in phantoms after amputation for trauma or in older people with arteriosclerosis, just as post-herpetic neuralgia and other pains are more distressing in later years.

It was soon realized that, while conspicuous disagreeable features were rare, for not more than two per cent. of patients complained seriously of pain, the more pleasant tingling, which almost always appeared, constituted the fundamentally important part of the phantom. Its constancy and uniformity strongly suggested an organic basis, with allowance being made for individual variation in obtrusiveness and strength, reflecting, no doubt, normal differences in the degree of cerebral activity. The motor manifestations were thought to be even more characteristically organic because of the objective evidence of sensori-motor reflex activity in the associated muscle contractions. It is important to recognize their close relationship and to distinguish them clearly from other more incidental features. We refer to these basic phenomena as the " natural phantom." It is characteristically mild and patients quickly get used to it so that it is rarely, if ever, a cause for complaint and never requires treatment.

All who have studied phantom limbs have naturally been concerned with determining the physiological level in the nervous system to which the existence of the phantom is to be attributed. We feel no obligation to combat the view, once widely held, that it is a purely hysterical manifestation, but we wish to point out that the features which distinguish the phantom from frankly psychotic hallucinations are found in that component which has been designated the natural phantom.

The manifestations of the natural phantom have been sufficiently described and the speculations to which they give rise already referred to. Here it is only necessary to stress the constancy of its appearance, the stereotyped form which it assumes, and especially the fact that the most vividly perceived and enduring parts are precisely those with the greatest cortical representation. We believe that its anatomical substrate is in the sensori-motor cortex.
The tingling is a positive sensation which presumably reflects increased activity in the sensory cortex. Its behaviour and relation to the factors which influence it suggest that it is more likely to originate spontaneously in the cells of the cortex than to be secondary to afferent impulses from the divided nerves in the stump. The main reasons for regarding it as a cerebral rather than a peripheral nerve phenomenon are: (1) The distribution of sensory tingling and of voluntary movement correspond with the extent of their respective cortical representation, and not to the anatomical distribution of a peripheral nerve. (2) The close functional correlation between sensation and willed movement. (3) The factors which intensify or induce the phantom arise from all levels of nervous activity. There is no evidence that the natural phantom is influenced by mechanical stimulation of the divided nerves, though the sensation thereby induced is correctly orientated. Were the tingling peripheral in origin and due to stimulation of the sensory cortex by constant mild impulses from the stump (and it may be), it could hardly be induced by central reflexes unless the cortex were in a state of heightened activity. Strong pain impulses from a traumatized stump submerge the phantom, just as happens when cerebral activity is diverted in other ways. (4) The phenomenon of telescoping, though not explained, is at least comprehensible if regarded as an expression of the slowly progressive obliteration of parts according to their cortical representation. Telescoping is a main argument in support of a cortical basis for the phantom, for it is perhaps the most complicated phenomenon and could hardly result from changes at a lower level. It reveals the integration of all influences that enter into the phantom-complex from those psychogenically determined at the highest levels to the simplest organic stimulus from a stump neuroma, and, as it were, fits them in to the final common path-the size and shape of the phantom. (5) As already indicated, it is among the varied phenomena of willed movements, rather than the sensory impressions, that the most suggestive evidence of cortical activity is found, and insufficient attention has been given to this feature in the past.

It seems as if immediately after amputation the related sensory cortex is temporarily in a state of increased activity which, however, gradually decreases during the following months. At first this activity is so strong that the phantom appears spontaneously in consciousness, whereas positive appreciation of a normal limb occurs only when a stimulus is received from the periphery. The related cortical field is, as it were, in a more receptive state than the remainder of the cerebral cortex and 
can respond to a variety of nervous impulses. Later, as the hyperexcitability subsides, the tingling ceases to appear spontaneously in consciousness, but still does so in response to reflex stimulation. Finally, the stronger inducing agents, or even direct stimulation of sensory fibres in the stump, fail to evoke any conscious sensation of the amputated part. Recently we observed, in a different connexion, a case which illustrates this final stage.

The patient was a woman from whom one of us removed a spinal neurofibroma attached to the seventh cervical root. Twelve years previously the index finger had been amputated at the proximal interphalangeal joint, following which she noticed the phantom tingling for the next two or three years but not afterwards. During recent weeks the neuroma had produced pins and needles in the thumb, middle, and ring fingers, but no sensation whatever was referred to the index. Transient, more severe post-operative pains had the same distribution. It may be assumed that the fibres from the stump of the index were stimulated in the root, as were those from the neighbouring digits, and it is interesting that the sensory cortex subserving the index finger, at first more active than the rest, eventually ceased to respond to stimuli from its afferent field.

Variability of cortical excitability and its eventual disappearance lead to the subject of subconscious cerebral activity. Although the phantom is essentially a conscious phenomenon, we have frequently referred to features which may be regarded as evidence of nervous activity at a subconscious level. For example, the gaps in an untelescoped phantom, which yet behaves as if it were complete, must still be represented centrally although not consciously perceived ; for otherwise, in the late stages of a distal amputation, it would not be possible to move the digits (which requires sensory guidance) if they were not still represented subconsciously. This, presumably, merely reflects normal subconscious cerebral activity. As the phantom weakens the decline of cerebral activity, shown at first by the cessation of conscious tingling, seems to go further, and in time the subconscious activity also fades out, as indicated by the disappearance of the gaps and of voluntary movements. We have thus been led to think, though not without hesitation, of activity at conscious and subconscious levels, and to regard the phantom as having truly disappeared only after the extinction of the latter phase. Only thus can we explain the difference between mere absence of positive sensation in a gap and the subsequent disappearance of the gap in the telescoped phantom.

The threshold of cortical excitability, being a functional and not an anatomical affair, is not uniform for the whole limb but is related to the relative degree of central representation of its parts. Increased excitability in relation to the fingers may persist after total disappearance of even subconscious representation of the weaker segments, giving the impression of a " baby arm with a large hand" and eventually of complete telescoping. One interesting feature of telescoping is that a segment, or any part of a limb, does not suddenly disappear but decreases in size gradually and evenly, a process which perhaps gives an inkling of the extremely complex neuronal process involved.

Our observations appear to indicate that the functional changes in the brain (and possibly also spinal cord) following amputation are limited to the centres associated with the missing part, except, $\stackrel{\triangle}{\triangle}$ it would seem, for a slight secondary involvement of of centres for neighbouring structures in the stump $\dot{\vec{\nabla}}$ (increased tone, sweating). These fields of altered excitability or function are affected differently by $\vec{\omega}$ the various phantom-inducing stimuli according to their origin, for example, the effect of willed movement does not spread to another phantom, whereas ? micturition affects both of lower limb phantomso $\overrightarrow{-}$ but not upper, while a severe stimulus like the shock of falling affects any phantom. It seems there is n\& $\infty$ widespread disturbance of cerebral activity althougi its general intensity appears to influence the strengt and possibly the rate of disappearance of the 3 phantom and therefore perhaps may account fof individual variation in these properties.

There can be little doubt that the central neuroni connexions subserving sensory and motor functions between different parts of a limb continue to maintain their functions after amputation, both within the phantom and between it and the stump. Those who have stressed the importance of peri- $\frac{\circ}{\Phi}$ pheral irritation in generating the phantom point $\stackrel{\varrho}{\rightarrow}$ out that the phantom follows the moving stump. This movement of the phantom, however, depends simply on the interpretation of proprioceptive impulses arising in the muscles of the stump. In fact, it supports the hypothesis of the central theory, for it shows that afferent impulses from the parts yet remaining (the stump muscles) are still in $\frac{5}{3}$ functional relationship with that part of the cortex in which resides the substrate of a consciously $\frac{0}{3}$ perceived gap between the phantom digits and the

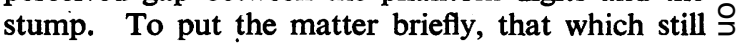
exists is working in harmony with that which has $\frac{D}{2}$ ceased to exist except as a pattern in the cortex.

The analysis of central neuronic connexions may $N$ be pushed to a more speculative stage concerning activity at conscious and subconscious levels. It $N$ is easy to conceive that during stump movements $\omega$ when there is little or no gap in the phantom (as 
after wrist disarticulation), the muscle afferents can maintain their neuronic connexions with the centres for the distal part of the phantom so that the stump and phantom move as a whole, for this is merely the normal proprioceptive mechanism when moving a rigidly held limb at a proximal joint. When there is a large gap in the phantom, including intervening joints, as between digits and shoulder, this connexion is still maintained, again, at a subconscious level, as in the normal limb. Later, when the subconscious gap has disappeared, functional connexion still remains between the stump muscles and the fully telescoped digits.

The continued harmony between centres is intimately bound up with the shape and size of the phantom and with telescoping. The original connexion between the digits and the stump may be by a long direct pathway or by short intersegmented relays. In either case the final link, after the intervening parts have faded out, is presumably direct. The digits, the leading part of the limb, thus continue to work with the stump, or perhaps with the girdle muscles, after the subsidiary supporting segments have disappeared.

This brings us to the extraordinary and probably very important phenomenon of the final inclusion of stronger digits within the normal anatomy of the stump, in which they may still be identified and moved voluntarily. This may also occur momentarily when the stump touches a solid object. The reference to the stump of the sensation aroused by stimulating a neuroma, and the sensation which runs down the stump during micturition, swallowing, etc., are probably of a similar nature. These represent a kind of extended final stage of telescoping and may be related to the mechanism known to neuro-physiologists as deviation of response. It may be that the cortex associated with the normal stump structures takes over the function of the abnormal digital cortex. Even more direct evidence pointing in the same direction is the transfer, after the phantom has disappeared, of the sensation on stimulating digital sensory fibres in a neuroma (which remain sensitive) to the normal skin of the stump.

We have assumed that the phantom is essentially a central phenomenon and have stated what seems to happen in terms of change in excitability and threshold differences, but we are still ignorant of the actual physiological processes involved or of their ultimate significance. The only obvious effect of amputation is division of the nerves, and if it is assumed that the phantom does not result from persistent irritation of nerve ends it follows that we must look for the cause in the isolation of central mechanisms from their natural peripheral connexion.
The phantom shows evidence of nervous activity at all levels. On the motor side can be seen the effect of the normal decreasing voluntary control of limb muscles from the most voluntary in the distal part to the most automatic at the proximal ; while the sensory component shows evidence of the lability of the cortex, a subject recently discussed by Walshe (1943). Lability of the cortex is further suggested by the final inclusion of the digits inside the stump, and even more directly by the transfer after the phantom has disappeared, of the sensation on stimulating digital sensory fibres to the normal skin on the stump.

The foregoing remarks suggest that the natural phantom depends upon changes in the spontaneous activity or threshold in the cortex following the loss of those impulses it normally receives from the amputated part. The " sensation" (or the absence of a conscious sensation) of a normal limb (for instance, a hand) quite likely depends upon the balanced integration of a multitude of impulses of all kinds constantly coming from many sources including the part of the limb representing the stump (for example, concerning weight and balance). It may be that the conscious appreciation of phantom tingling results (to adopt a suggestion made by Walshe (1942) in relation to other forms of disturbed nervous function) from the centres becoming unbalanced by the absence of normally opposing activities, or by liberation from a controlling influence (which would be from the missing part). We think that disturbance in the normal interaction between impulses from distal and proximal parts of the limb is important, and it may be that girdle structures are necessary for deviation of response. There is no evidence that the change goes beyond the limb, and the complete absence of impulses from a limb after spinal transection may account for the absence of a phantom in spinal lesions.

On the motor side, not only are the main movements of the hand consciously appreciated, but also the weaker secondary and tertiary contraction in proximal muscles. It is likely, however, that the contractions of the latter muscles (which are unmasked after amputation) occur normally during purposive movements of the limb though they are obscured by the more obvious contraction of the stronger supporting postural muscles.

The increased tone in the stump muscles evidently results from increased central excitability and is reminiscent of a release phenomenon. The leading part of the limb is an organ of movement and exploration acting under a high degree of voluntary direction which controls in some degree the contractions and possibly the tone of the more proximal 
supporting muscles. It is thus perhaps permissible to assume that when this control from the digital area is lost, as after amputation, the tone in proximal muscle groups is necessarily altered. Though this alteration in tone is the result of stimulation from the disturbed and hyperactive cortical areas, it appears to be a release from peripheral control rather than from higher centres as occurs in pyramidal lesions. The later diminution in cortical excitability would account for the ultimate reversion to more normal tone.

If it is assumed that the phantom reflects mechanisms in cerebral physiology then the motor manifestations show clearly a number of interesting features which have been described already and need only be recapitulated : (1) the close association between the motor and sensory cortex, which in fact function as one, and the primary importance of sensation in initiating voluntary movement ; (2) the distinction between the more voluntary and the less voluntary movements in relation to their sensory control and the different types of muscle fibres concerned; (3) an interesting motor mechanism which can activate the stump muscles in the absence of sensory guidance without, however, initiating movements in the phantom.

It has been seen that the only movements of which the phantom fingers are capable are flexion and to a lesser degree extension. If the central neuronic processes subserving more individual digit movement are intact, why, it may be asked, is the patient unable to perform such highly voluntary movements as abduction and adduction of the digits. Some insight into this anomaly is afforded from the fact that though such movements are highly voluntary, they are quantitatively weak by comparison with general finger flexion. In this connexion it may be noted that finger movements on cortical stimulation (Penfield and Boldrey, 1937) are limited to flexion and extension, and this too is what is seen clinically in epileptic movements.

There are, however, difficulties in accepting denervation, important though it appears, as the sole cause of the changes in the cortex. For example, we have not encountered a phantom in denervated digits after peripheral nerve injuries, in spinal lesions, or in gangrene. It may be that in these circumstances a phantom would be in conflict with sensations arriving at the cortex, not from the digits, but about them. It is, for example, possible that the slightest impression of weight from the insensitive part by pulling through tendons, joint capsules, etc., even though they are dead as in frost-bite, is enough to inform the cortex of the true state of affairs. An example of such inhibition of a phantom mechanism, on the motor side, by extraneous normal sensations with which it would $\underline{ }$ be in conflict, is seen in the absence of toe move- $z$ ment and twitching of thigh muscles when a belowknee stump is completely denervated.

We have never encountered a true phantom after peripheral nerve injuries, such as complete lesions of the sciatic nerve with denervation of the toes and ${ }_{\infty}$ forefoot, or of the brachial plexus with an insensitive hand. It is just possible, though unlikely, that some impulses are conveyed from these somatically- $\vec{z}$ denervated areas by sympathetic fibres accompanying the blood vessels. Patients with complete plexus $\stackrel{5}{\rightarrow}$ lesion may say they occasionally feel the fingers, $\frac{}{c}$ but this seems to be the momentary pins and needles $\underline{\underline{D}}$ due to stimulation of a neuroma and is, in reality, $\frac{\bar{D}}{\vec{D}}$ Tinel's sign. The digits to which this sensation is $\stackrel{\mathbb{Q}}{2}$ referred never show the phenomenon of telescoping. is We were never satisfied that true phantom tingling $\overrightarrow{0}$ appeared in totally denervated digits which, so far. as their intrinsic nerve supply is concerned, might $\vec{\omega}$ just as well have been amputated.

It would seem from the behaviour of phantoms that the knowledge that a limb is missing has little? or no importance in their genesis. Conversely, it $\overrightarrow{-}$ is unlikely that the known presence of an insensitive is part would prevent the appearance of phantom-like sensations in relation to it. The only appareni 을 explanation is that the presumed tendency for the cerebral cortex automatically to enter a phase of $z$ heightened activity in the absence of afferent stimu震 from the denervated part is inhibited by information $\overrightarrow{0}$ from the remainder of the limb. Such informatiof would indicate the continued presence of the insensitive part : there might, for example, be no alteration in weight or balance or in the tension of undivided long digital muscles.

True limb phantoms do not seem to occur after $\frac{\mathscr{Q}}{\mathbb{D}}$ complete spinal lesions, although over-anxious patients sometimes assert that they can feel the toes. $\overrightarrow{\overrightarrow{0}}$ We have had no opportunity to observe the effect 3 of a permanent spinal lesion or of spinal anæsthesia on lower limb phantoms. When the limbs are normal, spinal anæsthesia produces a transitory tingling as sensation is disappearing which is apparently similar to that in phantoms, but, as the $\frac{5}{3}$ anæsthesia becomes complete, the tingling disappears and is replaced by a feeling of heaviness $\delta$ of the legs. It thus appears that simple isolation of a limb from the sensori-motor cortex cannot $\frac{\text { ? }}{3}$ alone generate a phantom and that an additional $D$ factor is necessary, coming either from the girdle structures at the base of the limb (disturbance of $N$ balance, etc.) or from the ends of the divided nerves. We saw recently a man with double thigh amputation 0 in whom an inflammatory lesion in the upper dorsal $\omega$ spine; producing complete motor and sensory loss 
below the level, abolished the sensation of the phantom toes and the ability to move them. A few weeks later, however, as the lesion gradually subsided, the phantoms returned along with recovery of sensation on the trunk and movement of the stumps.

In regard to the occasional accompaniments which have already been discussed and illustrated, we have come to regard them as a reflection of individual peculiarity and not as an essential .part of the phantom, for they represent (a) a psychologicallyimportant experience in a normal person, $(b)$ an outlet for a psychopathic personality, or (c) a fixation object for a compensation neurosis. The more we saw of these manifestations, the more impressed we became with their similarity to hysteria, and it was interesting to see how the organic features of the natural phantom might be suppressed, as in hysterical anæsthesia or paralysis, or perverted into pain or involuntary movements. When one considers the complex and highly organized nature of many of these sensations, it becomes evident that they cannot result from stimulation of divided nerve fibres ; there seems, in fact, to be no alternative to the supposition that they originate at a high level in the nervous system.

We believe that the phantom portrays normal physiological mechanisms in the nervous system, and may, in fact, provide an avenue for their study. This belief in the organic behaviour of the natural phantom is strengthened by observations and conclusions made in totally different circumstances, namely: (1) the clinical phenomena of motor convulsions in epilepsy so analytically studied by Hughlings Jackson (1931), who pointed out the varying degree of voluntary control of different muscle groups, and the cortical representation of movements rather than of muscles ; (2) the work of Penfield and Boldrey (1937) on cortical stimulation in man, especially as regards representation in the sensory cortex ; (3) the primary importance of sensation in initiating movements shown by Mott and Sherrington (1895) in the deafferented monkey's limb, an experiment which had the effect of abolishing movements of the distal part of the limb while those depending on the proximal (less voluntary) muscles were much less impaired.

In peripheral nerve injuries we have been impressed, in cases with partial recovery, by the exactly similar distribution of grades of recovery, both sensory and motor, to that of the tingling and movement in phantoms, though it is, of course, reversed. This agrees with the accepted view that the final stages of complete recovery after nerve injuries depend on cortical integration, which, in turn, requires perfect peripheral regeneration.
When this is lacking, as in partial recovery, the more highly represented distal muscular and sensory functions lag far behind those of the more proximal structures.

In treatment it must first be decided to which group of symptoms (natural phantom, neuroma irritation, causalgia, or mental accompaniments) the patient's complaints belong, for they are sometimes interwoven and require careful analysis. In cases with severe mental manifestations an attempt should be made to discover the underlying cause, such as domestic worry (which precipitated the symptoms in several of our cases), unsettled compensation, or occasionally a latent psychosis. It is unnecessary to attempt altering the course of the natural tingling, about which the patient may complain in the early weeks during the initial disappointment of unexpectedly losing the limb. Knowing that this part of the phantom subsides sufficiently to be unnoticed after, say, six to eighteen months, the patient should, if necessary, be told it will weaken in the course of months, without, however, fixing any time limit.

We have no experience of attempting to relieve the more serious painful manifestation by spinal, cerebral, or sympathetic operations, though good results have been reported by others. 'It was found, however, that some patients are in such an impressionable state after past disappointments following inadequate operations, that even a peripheral operation, if satisfactory, is followed by complete suppression of the whole phantom. Such disappearance of symptoms, which may affect both natural phantom and overlay manifestations, can hardly depend on any " organic" change and must make us cautious in assessing the results of central operations.

\section{SUMMARY}

1. A study of some three hundred cases of amputation showed that a phantom limb is a natural and almost universal sequel to amputation.

2. Three kinds of - sensory phenomena may appear; (a) mild tingling, which forms the basic part of the phantom in nearly every case and probably arises in the cerebral cortex ; $(b)$ stronger pins and needles induced momentarily by touching a neuroma in the stump ; (c) occasionally, superadded sensations which may be disagreeable and painful and probably have a psychogenic basis. Rarely, a syndrome resembling causalgia is seen.

3. Special attention has been given to what is termed the " natural phantom" by which is meant the basic sensory tingling, its motor counterpart, the impression of willed movements, and other 
features. The spontaneous tingling appears in those parts (chiefly thumb, index, and great toe) having the largest cortical representation, and the belief that it originates in the sensori-motor cortex is strengthened by its close functional relationship with the motor phenomena. The willed movements also are stereotyped, and are most developed in relation to leading parts of the limb; they are associated with observable contraction of the appropriate muscles in the stump, and their behaviour reflects the fundamental importance of sensation in initiating and controlling voluntary movements. The phantom can be induced and intensified by a variety of reflex factors (for example, micturition) acting centrally.

4. The behaviour and evolution of the "natural phantom " is as if the related cerebral centres were for a time in a state of heightened activity, the later subsidence of which results in telescoping of the phantom and its eventual fading from consciousness, and perhaps also in the disappearance from subconscious levels of its neuronal patterns.

5. Unusual superadded sensations sometimes appear which are peculiar to the patient, and are believed to be psychogenically determined from the highest cerebral level. They may be mild, representing incidents in the patient's past experience, or, rarely, disagreeable - severe spontaneous pain, distorted attitudes, and involuntary spasmodic $\frac{\mathrm{C}}{\mathrm{O}}$ movements-dominating the phantom. The con- $z$ tinuation in the phantom of pre-amputation pain $\mathbb{Q}$ or posture seems to be determined, in many cases at least, by its psychological importance and not by its severity or duration.

6. The phantom cannot, therefore, be considered as a single entity for its manifestations may be derived from three levels in the nervous system $\overline{\bar{v}}$ (psychogenic, sensori-motor cortex, and peripheral nerve), and, before operating, it is important to determine to which of these the patient's complaints are most attributable.

7. Peripheral operations on the stump, apart from neurectomy for a sensitive neuroma, usually have no effect on the phantom. Occasionally in suggestible $\stackrel{\mathbb{D}}{\propto}$ patients and following a gratifying operation on the stump the phantom may be suppressed.

\section{REFERENCES}

Denny-Brown, D. E. (1929). Proc. roy. Soc. B, 104, 371. Hughlings Jackson, J. (1931). "Selected Writings." Edited by J. Taylor, and others. London.

Lhermitte, J. (1939). " L'image de notre corps." Paris. Mott, F. W., and Sherrington, C. S. (1895). Proc. royo Soc., 57, 481.

Penfield, W., and Boldrey, E. (1937). Brain, 60, 389.

Riddoch, G. (1941). Ibid., 64, 197.

Walshe, F. M. R. (1942). Ibid., 65, 48.

-_(1943). Ibid., 66, 104. 